ORIGINAL ARTICLES

\title{
Automatic bone drilling using a novel robot in orthopedic trauma surgery
}

\author{
Rumen Kastelov ${ }^{1}$, George Boiadjiev ${ }^{2,3}$, Tony Boiadjiev ${ }^{4}$, Kamen Delchev ${ }^{4}$, Kazimir Zagurski ${ }^{5}$, Boyko Gueorguiev*6 \\ ${ }^{1}$ Clinical Centre of Ministry of Domestic Affairs, Sofia, Bulgaria \\ ${ }^{2}$ Sofia University, Faculty of Mathematics and Informatics, Mechatronics, Robotics and Mechanics Dept, Sofia, Bulgaria \\ ${ }^{3}$ Bulgarian Academy of Sciences, Institute of Mechanics, Sofia, Bulgaria \\ ${ }^{4}$ Bulgarian Academy of Sciences, Institute of Information and Communication Technologies, Sofia, Bulgaria \\ ${ }^{5}$ Bulgarian Academy of Sciences, Institute of System Engineering and Robotics, Sofia, Bulgaria \\ ${ }^{6}$ AO Research Institute Davos, Davos, Switzerland
}

Received: June 30, 2017

DOI: $10.5430 /$ jbei.v3n2p62
Accepted: July 25, 2017

URL: https://doi.org/10.5430/jbei.v3n2p62

Online Published: August 1, 2017

\begin{abstract}
Background: Currently, applications of robotized systems in orthopedic trauma surgery steadily increase due to their functional abilities facilitating surgeon skills. The aim of this study is to present the functional advantages of a recently developed robot for bone drilling.

Methods: Ex-vivo experiments were performed on fresh porcine and bovine bones, as well as on fresh and embalmed human bones such as femora, vertebrae and rips, by the use of the robot for bone drilling to identify diverse control parameters and analyse thrust force and drill bit temperature during drilling.

Results: Experimental data during drilling is shown, control algorithms are described and bone drilling modes are characterized. Maximal values of thrust force and temperature are detected. Controlling thrust force seems to be the proper way to reduce force resistance and hence temperature during drilling. In automatic drilling mode, preliminary defined channel depth is drilled with accuracy of $0.1 \mathrm{~mm}$, and far cortex end recognition is proved to work reliably for automatic stop with minimal controlled soft tissue penetration. Cortex and bone marrow thickness are measured and analysed in real time.

Conclusion: The bone drilling robot is programmed to follow with high accuracy parameters defined by the surgeon. Enhancing surgeon's freedom and responsibility to make decisions, it can perform precise manipulations, decreasing the influence of subjective factors and increasing patient's safety.
\end{abstract}

Key Words: Automatic bone drilling, Overheating limitation, Robot, Thrust force control

\section{INTRODUCTION}

A variety of robotized systems have been developed during the last decade with applications facilitating diverse procedures in orthopedic trauma surgery. However, trauma and orthopedic surgeons still manipulate manually almost all types of drilling tools. Diverse bone drilling problems, encoun- tered by the surgeons prior to screw insertion, are extensively discussed in the literature. ${ }^{[1-5]}$ Yet, manual drilling tends to result in complications, affecting safety of patients treatment, such as larger apertures than needed, tendons or blood vessels damages, and overheating, often leading to bone necrosis. Correspondingly, stability of screw fixation and anchorage

\footnotetext{
*Correspondence: Boyko Gueorguiev; Email: boyko.gueorguiev@ aofoundation.org; Address: AO Research Institute Davos, Clavadelerstrasse 8, 7270 Davos, Switzerland.
} 
might be reduced and accompanied by delayed bone fracture healing as well as presence of infection-conductive necrotic tissue.

Several authors have discussed bone temperature dependence on the magnitude of axial force applied to the drill. ${ }^{[6-11]}$ Adding vibration during ultrasonically-assisted drilling of cortical bone was reported to reduce to some extent the resistant axial force. ${ }^{[12]}$ However, such drilling is still manually performed with vibrations at constant frequencies and amplitudes without any further control.

So far, attempts for temperature modelling and measurement during bone drilling have been undertaken by placing thermocouples inside the bone at known distances from the drilling area, however, with some indifferent or even contradictory outcomes. ${ }^{[7,8,11]}$

The need of high precision and accuracy of surgical manipulations can be successfully addressed by using robots for bone drilling applications in the orthopedic trauma surgery. ${ }^{[5]}$

The aim of this study is to present and discuss the func- tional abilities of the recently developed prototype Orthopedic Drilling Robot (ODRO) and the experimental evidence of its advantages with regard to automatic bone drilling. ${ }^{[13]}$

\section{MATERIALS AND METHODS}

Experimental approach is chosen in the current study for the sake of the complex matter of the object under investigation. The setup presented in some previous papers was further developed. ${ }^{[13-16]}$ Modelling was challenging to conduct since many parameters, including unknown ones, had to be taken into account. Yet, after performing a variety of experiments with fresh porcine and bovine bones, as well with fresh and embalmed human bones such as femora, vertebrae and rips, it was concluded that a robot for automatic bone drilling should incorporate components capable of achieving:

- Torque up to $1.5 \mathrm{Nm}$

- Force along the drill bit translation up to $100 \mathrm{~N}$

- Force sensor range up to $100 \mathrm{~N}$

- Drill temperature not over $52^{\circ} \mathrm{C}$

- Accuracy in drilling depth less or equal to $0.5 \mathrm{~mm}$

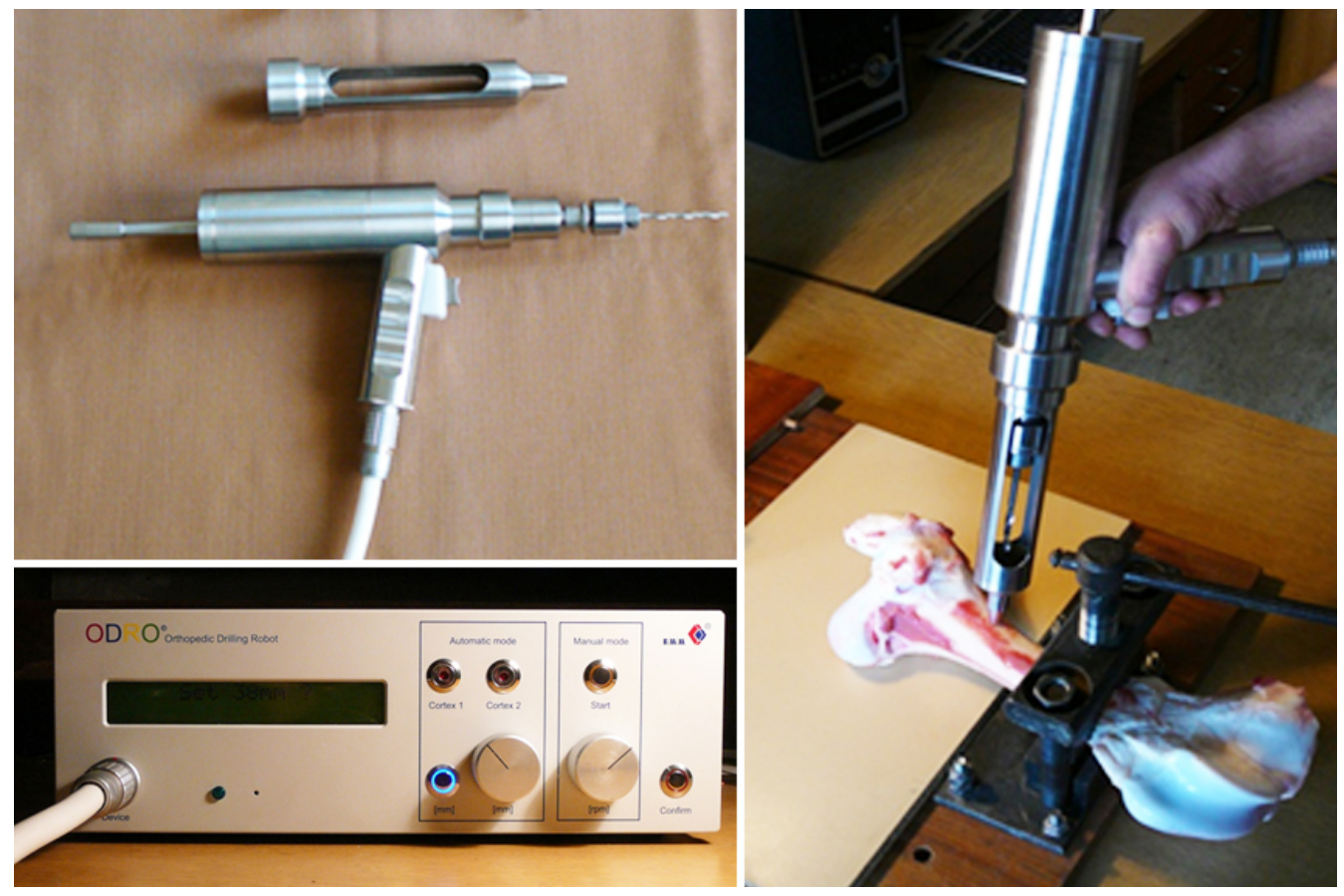

Figure 1. Executive module with accessories (top left) and control/power block (bottom left) of the ODRO together with a picture showing drilling execution (right)

The surgeon assures all the time mechanical contact between the executive module and the bone. The accessories help to maintain the chain of the module and the bone so that the program calculations from a reference point are possible. Sensor's and motors' controllers are inside the control block together with an electronic device to assure the interactions between them and the interface buttons and display. The surgeon holds the executive module keeping mechanical contact with the bone and pressing the start button. Drilling stops at button release. If the button is pressed again, the process execution continues from the point of interruption. If the surgeon does not release the button during the whole drilling procedure, the machine executes the task entirely and then the drill bit automatically returns to its initial reference point. 
In addition, the robot dimensions and mass needed to be minimized in the current design. The executive module with control and power blocks is shown in Figure 1. Two motors, namely a linear drive (stepper motor) and a brushless direct current (BLDC) motor, are installed inside the executive module together with a force sensor. The characteristics of the linear drive are voltage $5 \mathrm{~V}$, electrical power $7 \mathrm{~W}$ and maximal axial translation $105 \mathrm{~mm}$, whereas the BLDC motor is supplied with $50 \mathrm{~V}$ voltage and $200 \mathrm{~W}$ electrical power. The weight and the length of the executive module are $2 \mathrm{~kg}$ and $110 \mathrm{~mm}$, respectively. The robot is supplied with $220 \mathrm{~V}$ voltage. The cartridge van works with drill bits up to $8 \mathrm{~mm}$ diameter.

All control algorithms run in program environment Trinamic Motion Control Language (TMCL) and Integrated Development Environment (IDE). The commands can be executed in real time immediately after their input, or alternatively the whole program can be downloaded in the controller to perform autonomous execution of the commands.

In each software loop, the program recognizes the current state of the robot system, executes the corresponding algorithm and controls the transition to its next state according to preliminarily specified criteria.

The algorithm for current calculation of the next target position of the linear drive depends on the force sensor data. The actual force sensor data is continuously compared with previously values defined by safety requirements. The comparison is performed in order to check and keep the axial force within safety limits. An algorithm to identify and set as a bench mark the value of the resistant force at the beginning of the drilling process is also executed. ${ }^{[13,17]}$ The identified value, related to the bone characteristics and the drill bit diameter, is used to control later the instruction on whether to continue or to stop drilling.

\section{RESUlts}

Lots of effects, which occur during an automatic bone drilling regime and can be therefore registered only in the course of an experiment, are discussed. Temperature measurements during bicortical drilling throughout the entire bone tissue, performed under the same experimental conditions, resulted in maximal values of $50^{\circ} \mathrm{C}$ and $42^{\circ} \mathrm{C}$ for time intervals of 15 seconds in manual or 35 seconds in automatic drilling mode, correspondingly.

There is a great variability in the hardness of different bones in the human body due to age, gender, race or medical status of the patient. This will cause a consequent variation in the thrust force, torque and electrical power values of a particular drilling procedure. Because of that, the axial resistant force at the beginning of the drilling should be identified and registered for the specific bone object, taking into account patient's characteristics. ${ }^{[13]}$ The assessment of resistance force limits prevents a specific bone from possible crushing. The same approach is applied to control drilled hole's depth and recognise bone cortex thickness. When the cutting tip of the drill reaches the bone marrow, the force drops down. The resistant force is increased again when the drill bit starts drilling the far cortex.

The drilling mode considers the following monitored parameters:

- Time [s]

- Linear velocity $[\mathrm{mm} / \mathrm{s}]$

- Angular velocity [rpm]

- Resistant force [N]

- Temperature $\left[{ }^{\circ} \mathrm{C}\right.$ ]

\section{Discussion}

The functional abilities of the robot ODRO are discussed below.

\subsection{Drilling along one axis by keeping a straight line without transverse displacements}

A potential issue could be related to a possible displacement of the drill bit in the beginning of the drilling, or as a result of drill bit bending, especially at the far cortex. ${ }^{[16]}$ The surgeon assures and maintains a mechanical contact between the tip of the executive module and the bone surface, ensuring the necessary orientation of the drill bit prior to start of drilling (see Figure 1). The continual contact itself does not guarantee keeping a straight line without transverse displacements; that is surgeon's responsibility. However, the automatic drilling mode makes the manipulation easier in comparison to the manual one. This is realized by a four step algorithm, performed by the software as described below. After initial contact, in the first algorithm step the drilling starts with lower linear speed of $0.5 \mathrm{~mm} / \mathrm{s}$ to reach $1 \mathrm{~mm}$ depth with the aim to form a "centre" around the drill bit and prevent possible transverse displacements. This is very important especially when the drill bit orientation is not perpendicular to bone surface.

\subsection{Drilling without overheating}

Bone overheating depends mostly on inappropriate drilling force applied to the bone, drill bit sharpness and drilling time. Some inventors tried to avoid bone overheating by keeping high rotational speed (about 3,000 rpm) with their drilling devices, thus aiming to shorten the drilling time, whereas others reported possible prevention by application of low rotational 
speed, but with longer drilling times up to 2 minutes. ${ }^{[11]}$ In both cases the results were not satisfactory. Further, drilling regime with rotational speed 900-1,000 rpm and drilling time up to 16 s proved to be appropriate. ${ }^{[16,18]}$

In manual drilling mode with the robot acting as a standard power tool, the heating of the drilled bone completely depends on the surgeon's skills in handling the rotational speed, thrust force, drill bit orientation and drilling time.

In automatic drilling mode the drilling force control in the robot is performed by identification and setting of values for maximal speed and acceleration of the longitudinal translation motion in the controller. The next $1 \mathrm{~mm}$ depth of the drilling is executed at speed $2 \mathrm{~mm} / \mathrm{s}$ under algorithm step two control. Then bone density is assessed electronically and a specific value is obtained for its characterization. The aim of this identification is to control the applied drilling force. It is interesting to point out that in a previous study with ultrasonically-assisted drilling of cortical bone the thrust force was reduced from $60-65 \mathrm{~N}$ to $35-38 \mathrm{~N}$ by adding vibration ("back movement") during every drill turn. ${ }^{[12]}$ In the current work the algorithm for force control is with similar features, however, without implementation of "back movement". In case the thrust force increases above an identified level, the linear drive of the robot stops until the force, monitored by its sensor, drops down to this level because of the zero linear speed and the continuing drill rotation in the bone. Following, the linear drive is activated again. The algorithm calculates the next position of the drill bit edge based on the deviation of the actual resistant force from this specific value. The difference between the identified resistant force $\left(F_{R}\right)$ and the actual force $\left(\mathrm{F}_{\mathrm{Act}}\right)$ measured with the force sensor should actually be maintained as minimal as possible in both directions. This kind of force control during algorithm step three seems to be optimal in the sense of safety, however, it may lead to increase of the drilling time and higher drill temperature. That is why, a balance between these two parameters (applied force and drilling time) had to be found. After various experiments it was concluded that keeping the trust force $10 \%$ higher than $\mathrm{F}_{\mathrm{R}}$ limits the drilling time up to $16 \mathrm{~s}$, which is a very good compromise in the sense of drilling safety. ${ }^{[14-16,18,19]}$

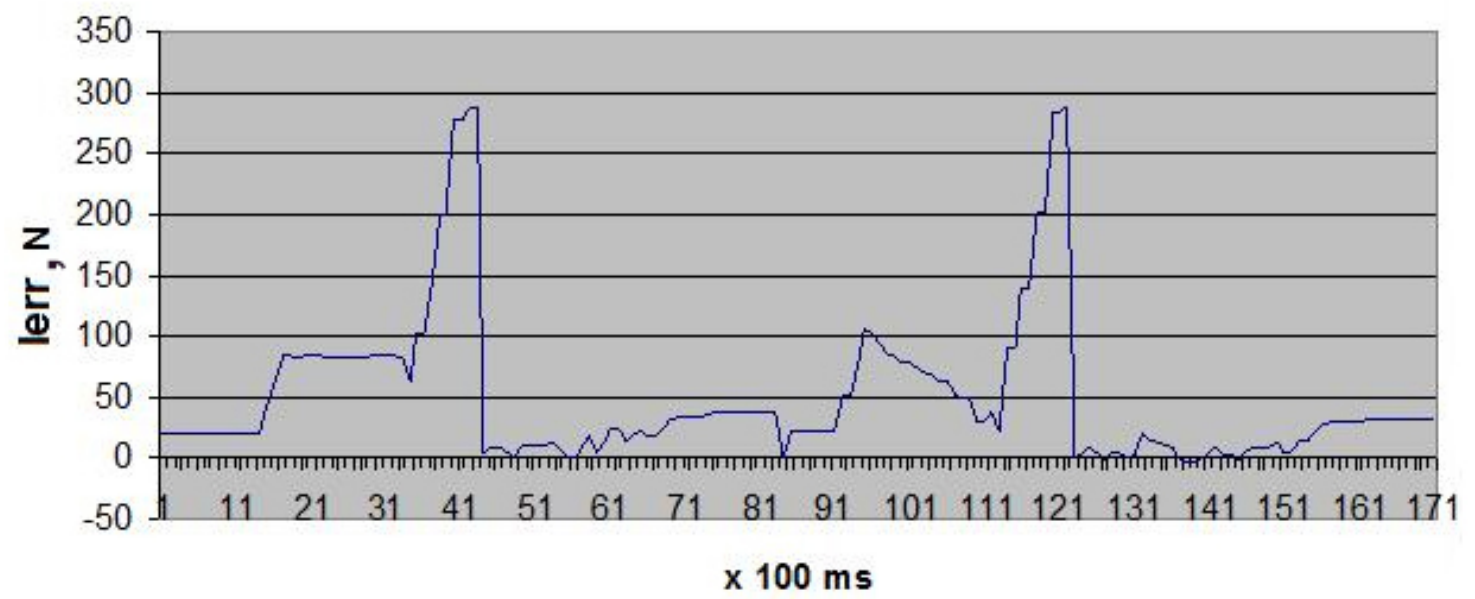

Figure 2. Experimental data for the integral component $\mathrm{I}_{\mathrm{err}}$ over time The horizontal axis shows time points recorded every $100 \mathrm{~ms}$ at a sampling rate of $10 \mathrm{~Hz}$. Drill bit diameter is $2 \mathrm{~mm}$.

\subsection{Drilling the whole bicortical bone with minimal pen- etration in soft tissue}

The decision for procedure end in automatic drilling mode is related to both preliminary set drilling depth or registration of far cortex outer surface and controlled in the fourth algorithm step. For this purpose, an integral component $\mathrm{I}_{\mathrm{err}}=$ $\sum_{1}^{4} \varepsilon$, where $\varepsilon=\mathrm{F}_{\text {Act }}-\mathrm{F}_{\mathrm{R}}$, is continuously calculated during drilling. A flow window with respect to the time, consisting of 4 samples of discretization, is considered to calculate continuously this integral component. For every flow window the software carries information for three previous samples values together with the temporary one. That calculation delivers information of the resistant force deviation near the front of drilling. The criterion to stop drilling is based on the parameters $F_{R}, F_{\text {Act }}, I_{\text {err }}$ and $F_{0}$, where $F_{0}$ is the free motion resistance. In addition, the drill bit is controlled to drill further the far cortex end with a reduced translational speed of $1 \mathrm{~mm} / \mathrm{s}$ at a certain depth to assure the so called "minimal penetration" in the soft tissue without widening the exit hole or breaking debris of the far cortex. Experimental timed data from calculation of $\mathrm{I}_{\text {err }}$ during porcine bone drilling are shown in Figure 2. Sampling rate and drill bit diameter are 
$10 \mathrm{~Hz}$ (data collected every $100 \mathrm{~ms}$ ) and $2 \mathrm{~mm}$, respectively. An interpretation of the drilling process with regard to the recorded time point numbers from Figure 2 is listed below as follows:

- Searching a contact with the near cortex surface: (time point numbers) 1-34

- Forming drill center and resistant force identification: $35-44$

- Near cortex drilling: 45-74

- Recognition of near cortex end and reduction of speed to $1 \mathrm{~mm} / \mathrm{s}: 70$

- Stop drilling: 71-73

- Stop motors: 74

- Manual start to continue drilling: 84

- Searching a contact with far cortex surface: 85-113

- Forming drill center and resistant force identification: 114-123

- Far cortex drilling: 124-163

- Recognition of far cortex end and reduction of speed to $1 \mathrm{~mm} / \mathrm{s}: 158$

- Stop drilling: $159-161$

- Stop motors: 162

- Drill bit back to initial reference position: after 163

The duration of the whole drilling process is $16 \mathrm{~s}$, including $4 \mathrm{~s}$ to drill the near and $5 \mathrm{~s}$ to drill the far cortex.

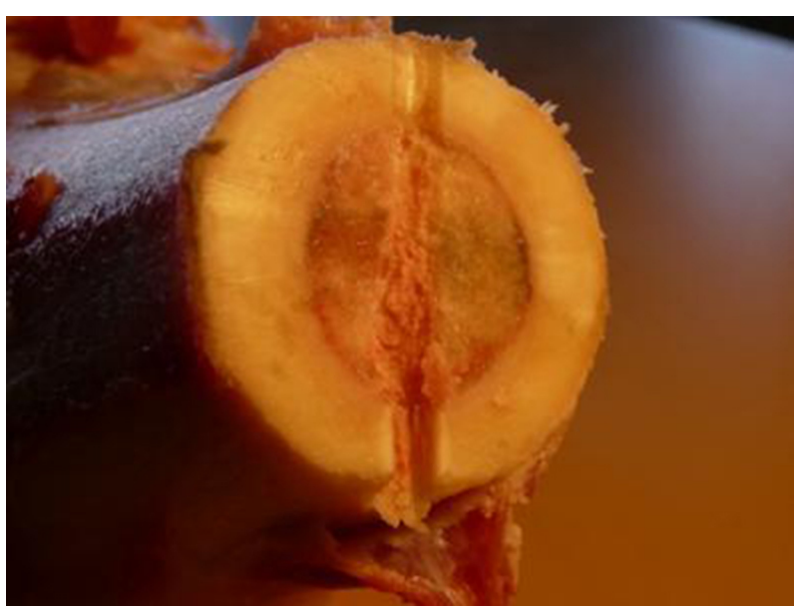

Figure 3. Exemplified porcine bone with longitudinal drill canal cross section after drilling

Drill bit diameter $6 \mathrm{~mm}$.

The time between cortex end recognition and stop of the drilling is $0.5 \mathrm{~s}$ (time point numbers $70-74$ and $158-162$ for the near and far cortex drilling, respectively). Taking into account the linear speed, the drill bit penetration out of bone far cortex is $0.7-1.0 \mathrm{~mm}$. A picture showing the drill canal in a longitudinal cross section after porcine bone drilling with a $6 \mathrm{~mm}$ drill bit is depicted in Figure 3.

\subsection{Drilling the near cortex only}

All four steps of the drilling algorithm described above (contact with bone surface, resistant force identification, drilling itself and automatic stop) are executed once. Finally, the drill bit is moved to its initial position and cortex thickness appears on the display.

\subsection{Information for drilling depth measurement in real time}

The current drilling depth is presented on the display so that the surgeon can control the drilling process in real time.

\subsection{Drilling of previously set depth}

In such a control mode the surgeon sets the desired depth, ranging from 2 to $200 \mathrm{~mm}$, at $1 \mathrm{~mm}$ step interval turning a potentiometer.

\subsection{Manual and automatic drilling modes}

The robot control can be selected in either automatic or manual drilling mode. In manual mode the drilling is performed in standard fashion adjusting the rotational speed with potentiometer. The automatic mode comprises three submodes: monocortical drilling of near cortex, bicortical drilling of both cortices (whole bone) or preliminary set depth drilling.

\subsection{Learning to work with the robot and how to use it} No special skills, exceeding the knowledge for orthopedic trauma surgery, would be necessary to use of the ODRO drilling robot. The different modes and submodes will be set by the surgeon using four buttons and a potentiometer. The respective information will be presented on the display with corresponding parameters. The selected mode is confirmed by pressing the "Confirm" button; the robot switches to operation mode after that.

\subsection{Comfortableness during drilling}

After setting the mode for a concrete drilling procedure, the surgeon needs to position and orient the executive module ensuring contact with the bone surface. Then the start button needs to be pressed. For normal functioning the surgeon needs to maintain the contact with the bone. The drill bit moves only when the surgeon keeps the start button pushed on. If the button is released, the robot immediately stops any motion. When the button is pressed again it continues with execution of the interrupted manipulation. If the surgeon decides to stop the procedure, he can set afterwards a new mode with a new task without restarting the system. The information on the display always shows which part of the task is executed up to the current moment, so that the surgeon can control the drilling in real time. For example, after procedure end in bicortical drilling mode the following information is 
displayed: near cortex thickness, bone marrow thickness, far cortex thickness and total drilling depth (see Figure 4).

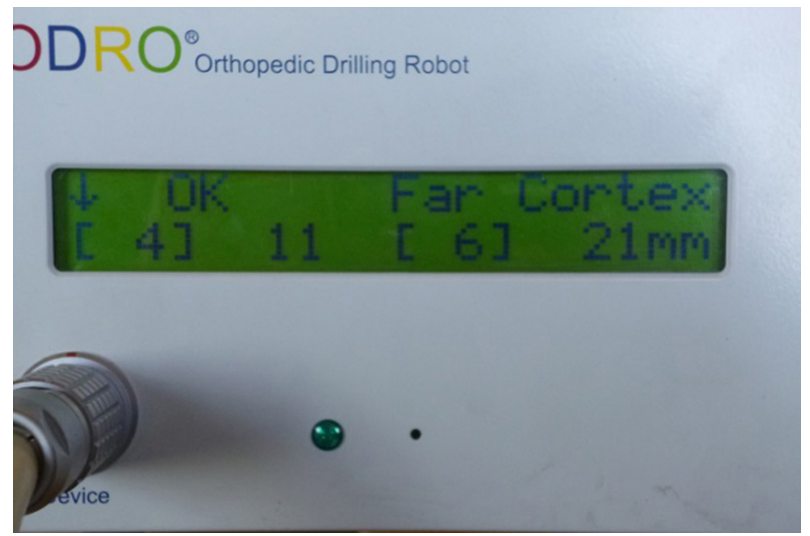

Figure 4. Exemplified information after procedure end of bicortical bone drilling

Near cortex thickness-4mm, bone marrow thickness-11 mm, far cortex thickness-6mm, total drilling depth-21mm.

Such information is very important during real operations because a whole conventional manipulation is eliminated, namely measurement of the currently reached drilled depth. During the automatic drilling, the depth is measured in real time with accuracy of $1 \mathrm{~mm}$, which has proven to be sufficient in the surgical practice.

\subsection{Sterilization}

The executive module, connection cable and power block have 30-pin LEMO connectors allowing disassembling, and are compatible with sterilization requirements. Buttons and potentiometers from hermetic bulging stainless steel are built in the power block. The cable length allows placement of the power block out of the sterile zone so that it just needs to be cleaned and disinfected. The executive module can be treated by chemical or high temperature sterilization. The cartridge and drill bit can also be dissembled and sterilized if necessary; the same applies for the robot accessories.

\subsection{Reliability and safety}

After switching on the electric power and pressing the start button, the robot firstly executes a self-test algorithm until sound signal indicates its end (the button needs to be pressed all the time during the procedure). This algorithm tests start button reliability (on/off), adjustment of the initial position of the cartridge with the drill bit, forward-and-backward translation within the working range, free motion resistance, algorithm steps and rotational motion.

The program implements criteria for right or wrong element functioning. If the test is successful, a confirmation message appears on the display and the robot switches to working mode, ready to set a concrete task. In case of a negative test the display shows "Self TEST ERR", the machine is blocked and cannot be further used.

Two parameters are set in automatic drilling mode: number of cortices to be drilled (one or two) and maximal cortex hole depth. The latter parameter limits the maximal cortex penetration, which prevents the patient from tissue damage in case of technical malfunction, i.e. the drill bit would not penetrate excessively in the surrounding tissue. This automatic function can be very helpful to the surgeon.

Another additional system function is monitoring of the agreement between set and current parameter values. If necessary, warning messages are displayed. For example, if the depth length is set too long and the drill bit length is not sufficient, the message "Error Prop" appears; then it is impossible to start the drilling with the selected drill bit. This check is performed immediately after contact to bone; if it is negative, the drill bit is moved to its initial position.

Another additional function is the drill bit sharpness control, which is based on a criterion for minimal depth necessary to be reached per unit of time in order to indicate possible lack of sharpness. In case of negative message a sound signal is released with a message "Drill Bit Err" shown on the display. However, the operation is not stopped automatically; the decision will be made by the surgeon.

\section{Conclusions}

A recently developed mobile and user-friendly prototype bone drilling robot for applications in orthopedic trauma surgery is presented. Its design and functions fill in the gap between the complex medical robotized systems with many degrees of freedom and the power tools for usual manual drilling. Its functional abilities are discussed, mainly paying attention to solving the problem with bone overheating, as well as assuring safety and accuracy during the drilling process. Using the drilling robot, the subjective assessment by the surgeon is not ignored as this is not necessary. The surgeon would still need to define the manipulation parameters, such as operating mode, drill bit selection and maximal penetration. The drilling robot is programmed to follow these parameters with high accuracy and keep them within critical limits. Its precise drilling performance seems to be advantageous for surgical applications, assuring higher reliability and safety during manipulation. We recommend the use of such kind of a robot in the orthopedic trauma surgery. ${ }^{[20]}$ Enhancing surgeon's freedom and responsibility to make decisions, it could autonomously perform precise manipulations, decreasing the influence of subjective factors and 
increasing patient's safety.

\section{Outlook}

A further version of the ODRO is under development with the aim to reduce its size and weight, and make it more comfortable for surgical applications. In the new mechanical construction the motors axes will be parallel and not collinear, thus scaling down the length of the device by one third. The weight will be reduced by $40 \%$ by using titanium alloy instead of stainless steel for production of some parts.

\section{ACKNOWLEDGEMENTS}

The authors are not compensated and there are no other institutional subsidies, corporate affiliations, or funding sources supporting this work unless clearly documented and disclosed. This investigation was performed with the assistance of BMM Ltd (Sofia, Bulgaria). Funding had no influence on the investigation including the results.

\section{REFERENCES}

[1] Lundskog J. Heat and bone tissue. An experimental investigation of the thermal properties of bone and threshold levels for thermal injury. Scandinavian Journal of Plastic and Reconstructive Surgery. 1972; 9: 1-80. PMid:4661159.

[2] Huiskes R. Some fundamental aspects of human joint replacement. Analyses of stresses and heat conduction in bone-prosthesis structures. Acta orthopaedica Scandinavica Supplementum. 1980; 185: 1208. https://doi.org/10.3109/ort.1980.51. suppl-185.0 1

[3] Wiggins KL, Malkin S. Drilling of bone. Journal of Biomechanics. 1976; 9: 553-9. https://doi.org/10.1016/0021-9290(76) 9 0095-6

[4] Clement H, Heidari N, Grechenig W, et al. Drilling, not a benign procedure: laboratory simulation of true drilling depth. Injury. 2012; 43: 950-2. PMid:22177726. https ://doi .org/10 .1016/j . inju ry. 2011.11 .017

[5] Rishi AM, Rutu PN. Detection of breakthrough during bone-drilling in orthopaedic surgery. International Journal for Technological Research In Engineering. 2014; 1: 794-8.

[6] Natali C, Ingle P, Dowell J. Orthopaedic bone drills-can they be improved? Temperature changes near the drilling face. The Journal of Bone and Joint Surgery British Volume. 1996; 78: 357-62. PMid:8636166.

[7] Lee J, Rabin Y, Ozdoganlar OB. A new thermal model for bone drilling with applications to orthopaedic surgery. Medical Engineering \& Physics. 2011; 33: 1234-44. PMid:21803638. https: //doi.org/10.1016/j.medengphy.2011.05.014

[8] Hillery MT, Shuaib I. Temperature effects in the drilling of human and bovine bone. Journal of Materials Processing Technology. 1999; 92-93: 302-8. https://doi.org/10.1016/S0924-0136(99)0 0155-7

[9] Moritz AR, Henriques FC. Studies of Thermal Injury: II. The Relative Importance of Time and Surface Temperature in the Causation of Cutaneous Burns. The American Journal of Pathology. 1947; 23: 695-720. PMid:19970955.

[10] Matthews LS, Green CA, Goldstein SA. The thermal effects of skeletal fixation-pin insertion in bone. The Journal of Bone and Joint Surgery American Volume. 1984; 66: 1077-83. PMid:6480636 https ://doi.org/10.2106/00004623-198466070-00015
[11] Augustin G, Zigman T, Davila S, et al. Cortical bone drilling and thermal osteonecrosis. Clinical Biomechanics (Bristol, Avon). 2012; 27: 313-25. PMid:22071428. https://doi.org/10.1016/j.cl inbiomech.2011.10.010

[12] Alam K, Mitrofanov AV, Silberschmidt VV. Experimental investigations of forces and torque in conventional and ultrasonically-assisted drilling of cortical bone. Medical Engineering \& Physics. 2011; 33: 234-9. PMid:21044856. https://doi.org/10.1016/j.medeng phy. 2010.10.003

[13] Boiadjiev G, Kastelov R, Boiadjiev T, et al. Design and performance study of an orthopaedic surgery robotized module for automatic bone drilling. The International Journal of Medical Robotics + Computer Assisted Surgery: MRCAS. 2013; 9: 455-63. PMid:23441029. https://doi.org/10.1002/rcs.1479

[14] Boiadjiev G, Boiadjiev T, Vitkov V, et al. Robotized System for Automation of the Drilling in the Orthopedic Surgery. Control Algorithms and Experimental Results. 9th IFAC International Symposium on Robot Control (SYROCO 2009). Gifu, Japan. 2009. p. 633-8.

[15] Boiadjiev T, Zagurski K, Boiadjiev G, et al. Identification of Bone Structure During Automatic Drilling in Orthopedic Surgery. Mechanics Based Design of Structures and Machines. 2011; 39: 285-302. https ://doi.org/10.1080/15397734.2011.550863

[16] Boiadjiev G, Delchev K, Boiadjiev T, et al. Automatic Bone Drilling in Orthopedic Surgery. Overcoming of the Drill Bit Bending at the Second Cortex. Applied Mechanics and Materials. 2014; 664: 41922. https://doi.org/10.4028/www.scientific.net/AMM. 6 64.419

[17] Boiadjiev T, Zagurski K, Boiadjiev G, et al. Automatic Bone Drilling in Orthopedic Surgery. Parameter Tuning of an Active Force Control. Applied Mechanics and Materials. 2014; 532: 208-11. https : //doi.org/10.4028/www.scientific.net/AMM.532.208

[18] Boiadjiev G, Delchev K, Boiadjiev T, et al. Controlled Trust Force Influence on Automatic Bone Drilling Parameters in the orthopedic surgery. International Journal of Pure and Applied Mathematics. 2013; 88: 577-92. https://doi.org/10.12732/ijpam.v88i4 .12

[19] Boiadjiev G, Kastelov R, Boiadjiev T, et al. Robot Application in Medicine for Orthopaedic Drilling Manipulation. In: Amerongen JV, Jonker B, editors. 8th Mechatronics Forum International Conference. Twente, Netherlands; 2002.

[20] Boiadjiev G. Orthopaedic Device. In: Bulgaria POoRo, editor. Bulgaria; 2011. 